\title{
Resonant-dynamics LTV feedforward for flexible motion systems
}

\author{
Nikolaos Kontaras ${ }^{1}$, Marcel Heertjes ${ }^{2}$, Hans Zwart ${ }^{3}$, and Maarten Steinbuch ${ }^{4}$
}

\begin{abstract}
Distributed parameter flexible systems face increasingly tighter specifications and performance requirements, which are typically handled by improving and developing novel feedforward control design methods. In the control synthesis of these systems, taking into account flexible dynamics play an increasingly important role. This work proposes an LTV feedforward control scheme which is based on the feasible and stable inversion of a minimum-phase fourth-order LTV approximation of the plant. This approximation takes into account resonant dynamics and (as a result) provides improved phase tracking. The results are validated through measurement results obtained through a rotational two-mass-spring-damper system.
\end{abstract}

\section{INTRODUCTION}

The ever-increasing requirements in the semiconductor industry in terms of increased throughput and smaller scales while retaining small servo errors lead to constant progress in terms of control design. In the current stage of evolution, a significant importance is attributed to feedforward control, since it constitutes the majority of the actuator control effort produced during scanning operation.

Traditional control schemes, e.g. classic acceleration feedforward schemes, account for the rigid body (RB) behavior of the plant. The subsequent development of snap feedforward [5] made it possible to account for the compliant and potentially resonant dynamics expressed by non-rigid-body (NRB) modes. Examples in the LTI domain include [3] which deals with the feedforward control of a motion stage system in the discrete-time domain, and [2] which compares different model-inversion based feedforward control designs for nonminimum-phase systems. In [1] and [8], a combination of feedforward and feedback control synthesis is used to account for flexible dynamics.

A fundamental aspect of stage systems used in lithography tools is the LTV nature it demonstrates during scanning, which is becoming increasingly more difficult to ignore, as the designs become more flexible especially when compared with the forces being applied to them [?] and the increasing

\footnotetext{
${ }^{1}$ Nikolaos Kontaras is with the Department of Mechanical Engineering, Control Systems Technology group, Eindhoven University of Technology, 5612 AZ Eindhoven, The Netherlands n.kontaras@tue.nl

${ }^{2}$ Marcel Heertjes is with ASML, Mechatronics Development, De Run 6501, 5504 DR Veldhoven, The Netherlands marcel.heertjeslasml.com

${ }^{3}$ Hans Zwart is with the Department of Mechanical Engineering, Dynamics and Control group, Eindhoven University of Technology, 5612 AZ Eindhoven, The Netherlands h.j.zwart@tue.nl, and with the Faculty of Electrical Engineering, Mathematics and Computer Science, Department of Applied Mathematics, University of Twente, 7500 AE Enschede, The Netherlands h.j.zwart autwente.nl

${ }^{4}$ Maarten Steinbuch is with the Department of Mechanical Engineering, Control Systems Technology group, Eindhoven University of Technology, 5612 AZ Eindhoven, The Netherlands m. steinbuch@tue.nl
}

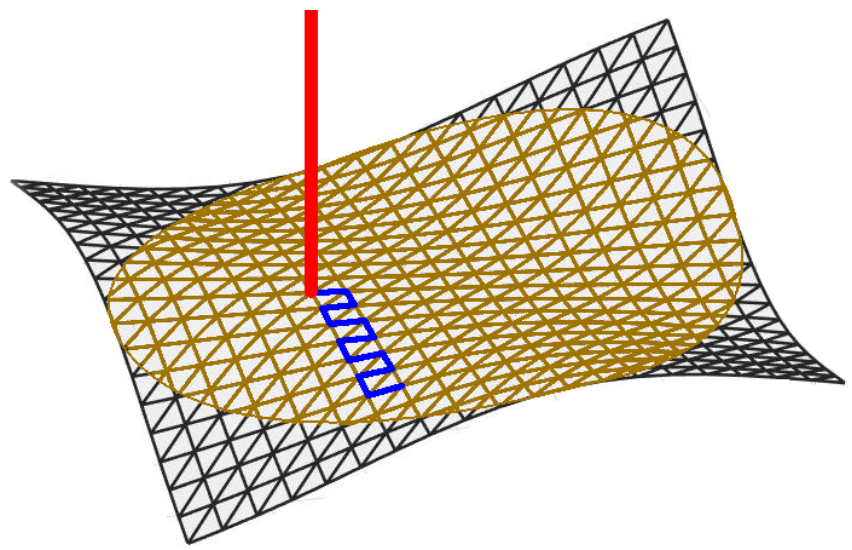

Fig. 1: Flexible thin plate representing a stage system. The point of interest changes with time (blue), inducing LTV dynamics.

accuracies required. This is illustrated by means of the thin plate shown in Fig. 1. It can be seen that the flexible dynamics of a thin plate, here representing a wafer stage, are different in each performance location. As the performance location changes with time, LTV dynamics determine the system's response. As such, there have been numerous works which attack the feedforward servo control problem in the LTV domain. One of the earlier works applicable to this framework, [4] uses stable inversion to calculate a non-causal feedforward signal. Specifically for LTV systems, the work in [7] finds that difficulties arise when the relative degree of the system changes during operation, highlighting also the issue of shifting from a minimum-phase to a non-minimumphase plant mid-experiment, i.e. during scanning. In [6], a lifted system representation is used to calculate the inverse model of an LTV plant in discrete-time. The work in [9] presents an LTV feedforward capable of accounting for timevarying compliant dynamics of flexible systems. Similar to previous works, it is shown that time-derivatives of the timevarying parameters of the plant need to be taken into account, signifying the understanding that LTV systems are more than simply the series connection of LTI systems. In fact, the manner, e.g. the speed, by which the time-variation takes place is key in achieving motion performance.

The work in this paper introduces a resonant and compliant dynamics LTV feedforward control scheme. The class of systems addressed is similar to [9], i.e. double-integratorbased flexible systems with position-dependent time-varying flexible dynamics. A first contribution in this work is that an LTV fourth-order model is used to approximate the total 
time-varying compliant dynamics of the plant, and due to its low damping coefficient, it can also account for the dominant resonant dynamics. The proposed control scheme is able to account for arbitrarily high time-varying dynamics, given appropriate smoothness requirements for the timevarying parameters. Moreover, in comparison with [9], the controller shows significantly smaller phase delay due to the low damping coefficient of the model approximation. Therefore, this control scheme can account for positiondependent flexible dynamics in high-precision motion stages, which traditionally suffer from internal deformations. As a second contribution, a global asymptotic stability criterion dedicated to the design of the feedforward controller is provided via a common quadratic Lyapunov function (CQLF) formulation, which serves as a hard constraint on the controller's performance. A third contribution involves measurement results, which serve as a proof of concept of the viability of the control design in the motion control practice, and which will be performed on a rotational two mass-springdamper system.

The remainder of this work is organized as follows. Section II poses the problem. Section III presents the proposed feedforward control scheme. Section IV investigates feasibility and stability aspects. Section V discusses measurement results in discrete time using a setup of a mass-spring-damper system. Finally, Section VI gives concluding remarks.

\section{Problem STATEMENT}

Consider the class of LTV systems illustrated in Fig. 2, which consists of one RB mode and an arbitrary amount of NRB modes post-multiplied by time-varying compliances, which serve as indicators of a time-varying sensor location. ${ }^{1}$

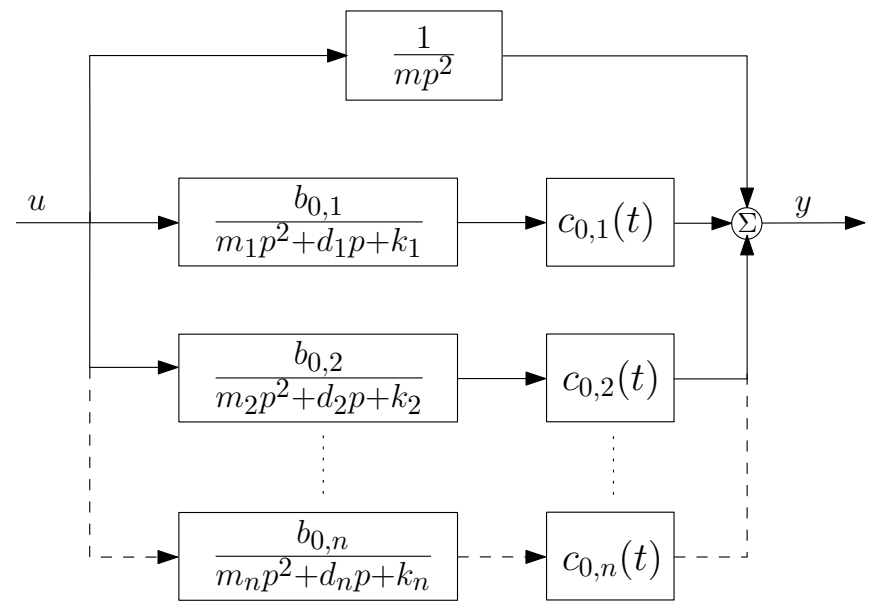

Fig. 2: Block diagram of LTV flexible system $\mathcal{H}$.

This scheme represents a lumped parameter system, or a finite-order approximation of a distributed parameter system, and can be described by the LTV state-space model,

$$
\mathcal{H}:\left\{\begin{array}{ll}
\dot{x}(t) & =A_{m} x(t)+B_{m} u(t) \\
y(t) & =C_{m}(t) x(t)
\end{array},\right.
$$

\footnotetext{
${ }^{1}$ The time-differential operator $p=\mathrm{d} / \mathrm{d} t$ is used, rather than using the Laplace variable $s$, to clearly distinguish between time and frequency.
}

where $A_{m} \in \mathbb{R}^{n \times n}, B_{m} \in \mathbb{R}^{n \times 1}, C_{m}(t) \in \mathbb{R}^{1 \times n}$, with $n \in \mathbb{N}$. The state and input matrices, $A_{m}$ and $B_{m}$ respectively, are constant-valued. Due to the aforementioned post-multiplication, only the output matrix $C_{m}(t)$ can be considered time-dependent.

As a special case of LTI, which is included in the class of (1), consider the single-input single-output (SISO) flexible system as the real plant to be accounted for via feedforward control, which for the purpose of presentation is limited to one RB and two NRB modes, as follows,

$\mathcal{H}(s)=\frac{1}{m s^{2}}+c_{1} \frac{\omega_{1}^{2}}{s^{2}+2 \omega_{1} \zeta_{1} s+\omega_{1}^{2}}+c_{2} \frac{\omega_{2}^{2}}{s^{2}+2 \omega_{2} \zeta_{2} s+\omega_{2}^{2}}$,

where $m$ is the mass, $c_{1}$ and $c_{2}$ the compliances of the two NRB modes, where the first mode is dominant, i.e. $c_{1} \gg$ $c_{2}$, located at frequencies $\omega_{1}$ and $\omega_{2}$, with $\omega_{2}>\omega_{1}$, and damping coefficients $\zeta_{1}$ and $\zeta_{2}$, respectively.

The control scheme in Fig. 3 is applied on $\mathcal{H}$ in (2), where a reference trajectory is given by $r_{y}$. The feedforward controller $\mathcal{C}_{f f}$ produces the signal $u_{f f}$, which takes into account the dynamics of $\mathcal{H}$. The feedback controller $\mathcal{C}_{f b}$ can be chosen appropriately with respect to the control objectives, external disturbances, and the plant $\mathcal{H}$ itself.

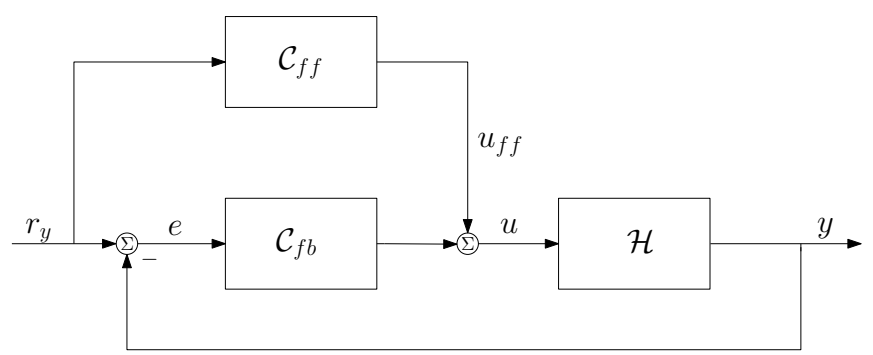

Fig. 3: Block diagram of the proposed LTV feedforward control scheme.

Now let us introduce the following fourth-order model $\mathcal{P}_{\mathrm{d}}$ can be used as an approximation of $\mathcal{H}(s)$ in (2),

$$
\mathcal{P}_{d}(s)=\frac{1}{m s^{2}}+\left(c_{1}+c_{2}\right) \frac{\omega_{s}^{2}}{s^{2}+2 \omega_{s} \zeta s+\omega_{s}^{2}},
$$

whose inverse serves as a basis for $\mathcal{C}_{f f}$, where $\omega_{s}$ is the cutoff frequency and $\zeta$ the damping coefficient. It can be seen that if the feedback controller $\mathcal{C}_{\mathrm{fb}}=0$, the resulting tracking error has the following sensitivity transfer function

$$
\mathcal{S}(s)=\frac{e(s)}{r(s)}=1-\mathcal{H}(s) \mathcal{P}_{d}(s)^{-1} .
$$

Consider two cases for (3), $\mathcal{P}_{d_{1}}$ where the NRB mode matches $\mathcal{H}$, i.e. $\omega_{s}=\omega_{1}$ and $\zeta=\zeta_{1}$, and $\mathcal{P}_{d_{2}}$ where the NRB does not match $\mathcal{H}$, and more specifically $\omega_{s}<\omega_{1}$ and $\zeta=1$ (only real poles are allowed), as in [9]. The frequency response functions of these two cases are depicted in Fig. 4. It can be seen that $\mathcal{P}_{d_{1}}$ is better able to match the phase of $\mathcal{H}$. The phase of $\mathcal{H}$ increases right before its resonance occurs, due to complex-valued poles, while $\mathcal{P}_{d_{2}}$ is losing phase due to its real poles. As such, the inverse of $\mathcal{P}_{d_{1}}$, if 


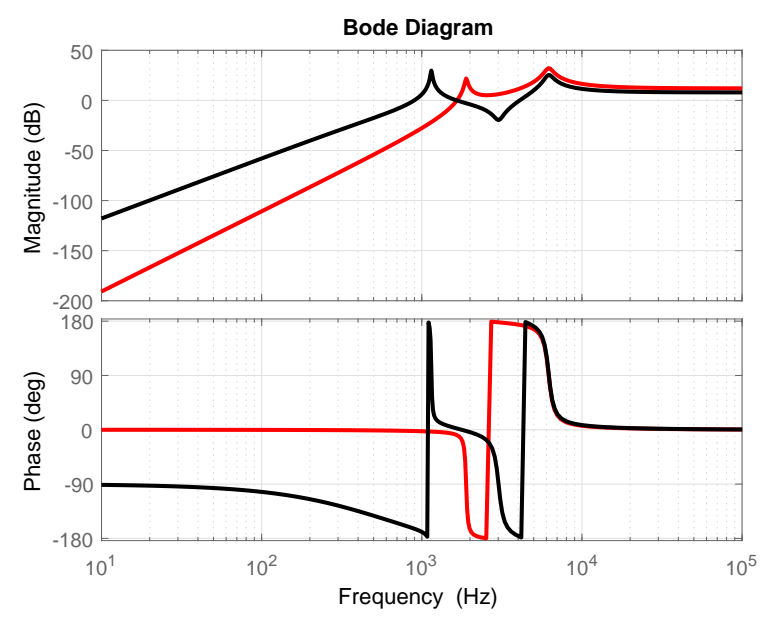

Fig. 4: Sensitivity frequency response functions $\mathcal{S}(s)$ using $\mathcal{P}_{d_{1}}(s)$ (black curve) and $\mathcal{P}_{d_{2}}(s)$ (red curve) .

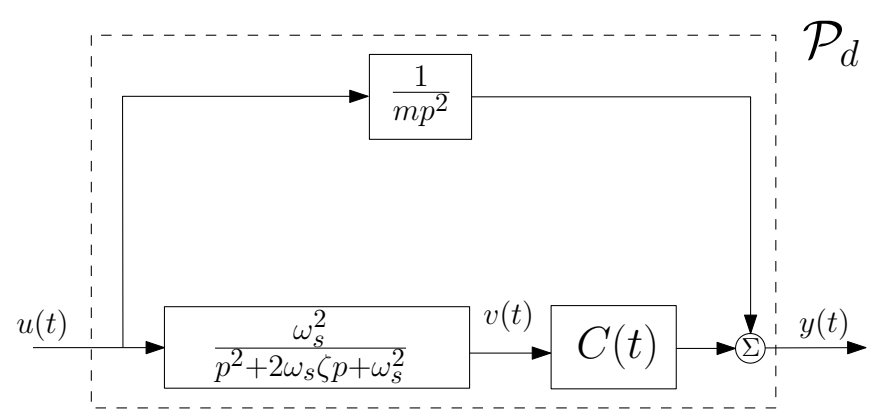

Fig. 5: Plant approximation $\mathcal{P}_{d}$ underpinning the proposed feedforward controller, consisting of one RB mode, and second-order low-pass filter cascaded with a time-varying gain $C(t)$ which equals the time-varying compliance of the system.

stable, is expected to provide better error suppression at the low-frequency range. Moreover, the resonance shifts toward a higher frequency which gives suppression over a larger frequency interval.

The aim of this work is to extend the concept represented by $\mathcal{P}_{d_{1}}^{-1}$ for the LTI system in (2), toward the LTV system in (1).

\section{RESONANT-DYNAMICS FEEDFORWARD CONTROL SCHEME}

For the LTV case, consider the plant approximation $\mathcal{P}_{d} \simeq \mathcal{H}$ illustrated by the block diagram in Fig. 5. The inverse of $\mathcal{P}_{d}$ forms the basis of $\mathcal{C}_{f f}$, which filters the desired trajectory $r_{y}$ in order to produce the feedforward signal $u_{f f}$. The lower branch of $\mathcal{P}_{d}$ can be perceived from the perspective of capturing not only the plant's compliant dynamics $C(t)$, but potentially also the resonance of a single NRB mode through the proposed low-pass filter. This would require that the second-order low-pass filter's poles are complex, such that it approaches or matches with the damping coefficient of the NRB mode.

The LTV model of $\mathcal{P}_{d}$ is governed by the equations

$$
\begin{aligned}
y(t) & =\frac{1}{m} \iint u(\tau) \mathrm{d} \tau+C(t) v(t) \Leftrightarrow \\
\frac{\mathrm{d}^{2}(m y(t))}{\mathrm{d} t^{2}} & =u(t)+\frac{\mathrm{d}^{2}(m C(t) v(t))}{\mathrm{d} t^{2}},
\end{aligned}
$$

and

$$
\omega_{s}^{2} u(t)=\ddot{v}(t)+2 \omega_{s} \zeta \dot{v}(t)+\omega_{s}^{2} v(t)
$$

where $\omega_{s}>0$ denotes the resonance frequency and $\zeta>0$ the damping coefficient. The function $C(t)=C\left(r_{p}(t)\right)$ gives the time-varying compliance of the plant $\mathcal{H}$. The timevarying parameter $r_{p}(t)$ indicates the manner by which the performance location changes over time. For stage systems this is usually a spatial variable, indicating the point of interest.

Given a desired trajectory $r_{y}(t) \in \mathcal{C}^{1}$, solving (6) with respect to $u(t)$ and after substitution into (5) gives

$$
\begin{aligned}
& \ddot{v}(t)=\underbrace{-\frac{2 \omega_{s}\left(\zeta+\omega_{s} m \dot{C}(t)\right)}{\omega_{s}^{2} m C(t)+1}}_{\xi_{1}(t)} \dot{v}(t) \underbrace{-\frac{\omega_{s}^{2}(1+m \ddot{C}(t))}{\omega_{s}^{2} m C(t)+1}}_{\xi_{2}(t)} v(t) \\
& \underbrace{+\frac{\omega_{s}^{2} m}{\omega_{s}^{2} m C(t)+1}}_{\xi_{3}(t)} \ddot{r}_{y}(t) .
\end{aligned}
$$

Equation (7) reveals that in order to prevent division by zero, it is required that,

$$
\omega_{s}^{2} m C(t)+1>0 .
$$

Since one control objective of this feedforward controller is to cancel the dominant resonance of the plant located at frequency $\omega_{1}[\mathrm{rad} / \mathrm{sec}]$, according to (8) the compliance function is lower-limited by,

$$
C(t)>\frac{-1}{m \omega_{1}^{2}} .
$$

If (9) cannot be satisfied, a choice has to be made of either accounting for the full compliance of the plant, or the resonant dynamics of the NRB mode corresponding to frequency $\omega_{1}$. In the latter case, the tracking error naturally correlates with the magnitude of negative compliance the feedforward controller was unable to account for. In the first case, a smaller error than [9] is expected, due to the lower damping coefficient which guarantees better phase tracking, as shown in Section II.

Equation (7) can be solved for the signals $v(t), \dot{v}(t)$, and $\ddot{v}(t)$ through numerical integration. The feedforward control input is given by

$$
u_{f f}(t)=\underbrace{\frac{1}{\omega_{s}^{2}}}_{\mu_{1}} \ddot{v}(t)+\underbrace{\frac{2 \zeta}{\omega_{s}}}_{\mu_{2}} \dot{v}(t)+v(t) .
$$

The second time-derivative of $r_{y}$ is assumed to be known a priori, which ensures the exact calculation of the feedforward 
signal $u_{f f}(t)$ for time $t \in \mathbb{R}_{\geq 0}$. A state-space realization of the feedforward controller is given by,

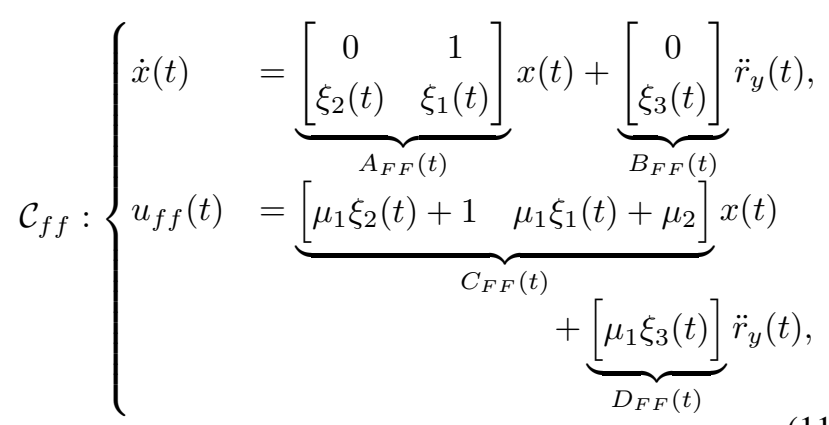

where $x(t)=\left[\begin{array}{ll}x_{1}(t) & x_{2}(t)\end{array}\right]^{T}=\left[\begin{array}{ll}v(t) & \dot{v}(t)\end{array}\right]^{T}$. The initial state of (11) is zero, as the system is assumed to be at rest for $t<0$. Successful model inversion ensures that this feedforward control scheme can successfully account for rigid body, compliant, and resonant dynamics. However, depending on the choices for the cut-off frequency $\omega_{s}$, compliance function $C\left(r_{p}(t)\right)$, and damping ratio $\zeta$, the feedforward signal can become unbounded, which potentially deteriorates performance.

\section{BOUNDED-INPUT BOUNDED-OUTPUT STABILITY}

Given the feasibility condition in (8), a feedforward signal can always be calculated. However, the performance associated with the controller is not guaranteed in the sense of a bounded-input bounded-output signal.

Bounded-input bounded-output stability of the LTV feedforward controller in (11) can be assessed in two steps. Step 1, guaranteeing asymptotic stability for the autonomous system

$$
\dot{x}(t)=A_{F F}(t) x(t),
$$

via an appropriate Lyapunov function $V(x)$, and step 2, requiring boundedness for $B_{F F}(t), C_{F F}(t)$, and $D_{F F}(t)$, guaranteeing bounded-input bounded-output (BIBO) stability for the non-autonomous system. For deriving bounds in step 2 , the reader will be referred to [9]. To the best knowledge of the authors, there are no necessary and sufficient stability conditions for arbitrary LTV systems that can be practically verified [10]. As such, in this paper, an additional theorem for finding a candidate Lyapunov function $V(x)$ for step 1 is presented. The benefit in doing so will be the increase of cases for which stability can be guaranteed.

Theorem 1. Consider the real-valued, second-order timevarying autonomous system

$$
\ddot{x}(t)-\xi_{1} \dot{x}(t)-\xi_{2} x(t)=0, \quad \forall t>t_{0},
$$

where $t_{0}$ is the initial time. The time-varying parameters,

$$
\xi_{1}=\xi_{1}(t), \quad \xi_{2}=\xi_{2}(t) \in \mathcal{C}^{2},
$$

are uniformly bounded from below and above as follows,

$$
\epsilon_{2} \leq \xi_{1}(t) \leq \epsilon_{1}<0
$$

and

$$
\epsilon_{4} \leq \xi_{2}(t) \leq \epsilon_{3}<0
$$

Define the time-varying functions

$$
\delta_{1}(\beta, t)=-\left(\beta^{2}+\beta \xi_{1}(t)+\xi_{2}(t)\right)+2 \sqrt{\beta\left(\beta+\xi_{1}(t)\right) \xi_{2}(t)}
$$

and

$$
\delta_{2}(\beta, t)=-\left(\beta^{2}+\beta \xi_{1}(t)+\xi_{2}(t)\right)-2 \sqrt{\beta\left(\beta+\xi_{1}(t)\right) \xi_{2}(t)} .
$$

A quadratic Lyapunov function which guarantees global exponential stability for system (13) exists if and only if there exists a $\beta$ satisfying

$$
0<\beta<\min \left(-\xi_{1}(t)\right), \quad \forall t>t_{0},
$$

such that an $\epsilon \in \mathbb{R}_{>0}$ can be found for which

$$
\max \delta_{2}(\beta, t)<\epsilon<\min \delta_{1}(\beta, t), \quad \forall t>t_{0} .
$$

Proof. Consider a candidate quadratic Lyapunov function, which without loss of generality can be written as

$$
V(x)=x^{T} P x=x^{T}\left[\begin{array}{cc}
\alpha & \beta \\
\beta & 1
\end{array}\right] x,
$$

where

$$
\alpha=\beta^{2}+\epsilon,
$$

for some $\epsilon \in \mathbb{R}_{>0}$. System (13) can be written in state-space form as,

$$
\dot{x}(t)=A_{F F}(t) x(t)=\left[\begin{array}{cc}
0 & 1 \\
\xi_{2} & \xi_{1}
\end{array}\right] x(t), \quad \forall t>t_{0} .
$$

The time derivative of (21), given system (23), reads

$$
\begin{aligned}
\dot{V}(x(t)) & =\dot{x}^{T} P x+x^{T} P \dot{x} \\
& =x^{T} A_{F F}(t)^{T} P x+x^{T} P A_{F F}(t) x \\
& =x^{T}\left(A_{F F}(t)^{T} P+P A_{F F}(t)\right) x \\
& =x^{T}\left[\begin{array}{cc}
2 \beta \xi_{2} & \alpha+\beta \xi_{1}+\xi_{2} \\
\alpha+\beta \xi_{1}+\xi_{2} & 2\left(\beta+\xi_{1}\right)
\end{array}\right] x \\
& =x^{T} \underbrace{\left[\begin{array}{cc}
2 \beta \xi_{2} & \beta^{2}+\beta \xi_{1}+\xi_{2}+\epsilon \\
\beta^{2}+\beta \xi_{1}+\xi_{2}+\epsilon & 2\left(\beta+\xi_{1}\right)
\end{array}\right]}_{P_{V}(t)} x
\end{aligned}
$$

The real-valued $\dot{V}(x(t))$ in (24) is negative for any $x \in \mathbb{R}_{\neq 0}$ if and only if $P_{V}(t) \prec 0$, which holds if and only if its first principal minor is negative and second principal minor positive. This requirement yields the following conditions,

$$
2 \beta \xi_{2}<0
$$

and

$$
4 \xi_{2} \beta\left(\beta+\xi_{1}\right)>\left(\beta^{2}+\beta \xi_{1}+\xi_{2}+\epsilon\right)^{2} .
$$


Condition (25) combined with (16) gives $\beta>0$. The right side of (26) is non-negative, thus it can be seen that we require

$$
4 \xi_{2} \beta\left(\beta+\xi_{1}\right)>0, \quad \forall t>t_{0},
$$

thus

$$
0<\beta<\min \left(-\xi_{1}(t)\right), \forall t>0 \text {. }
$$

Therefore the possible values of $\beta$ which can yield a feasible Lyapunov function are bounded from above and below. Returning to (26), the polynomial is expanded with respect to $\epsilon$ as follows,

$$
-\epsilon^{2}-2\left(\beta^{2}+\beta \xi_{1}+\xi_{2}\right) \epsilon-\left(\beta^{2}+\beta \xi_{1}-\xi_{2}\right)^{2}>0 .
$$

The second-order polynomial (29) has a negative second derivative with respect to $\epsilon$, thus in order to be positive for some $\epsilon$ it needs to have real roots. Its discriminant is required to be non-negative, or

$$
\begin{aligned}
\Delta & =\left(-2\left(\beta^{2}+\beta \xi_{1}+\xi_{2}\right)\right)^{2}-4(-1)\left(-\left(\beta^{2}+\beta \xi_{1}-\xi_{2}\right)^{2}\right) \\
& =16 \beta\left(\beta+\xi_{1}\right) \xi_{2} \geq 0
\end{aligned}
$$

Inequality (30) holds when (28) holds, thus it is automatically satisfied. The (real) roots of (29) are then given by,

$$
\delta_{1}(\beta, t)=-\left(\beta^{2}+\beta \xi_{1}(t)+\xi_{2}(t)\right)+2 \sqrt{\beta\left(\beta+\xi_{1}(t)\right) \xi_{2}(t)},
$$

and

$$
\delta_{2}(\beta, t)=-\left(\beta^{2}+\beta \xi_{1}(t)+\xi_{2}(t)\right)-2 \sqrt{\beta\left(\beta+\xi_{1}(t)\right) \xi_{2}(t)} .
$$

Thus, a common quadratic Lyapunov function for (13) exists if and only if an $\epsilon$ can be found such that

$$
\max \delta_{2}(\beta, t)<\epsilon<\min \delta_{1}(\beta, t), \quad \forall t>t_{0},
$$

which guarantees global exponential stability.

Stability using Theorem 1 can be practically utilized by plotting (31) and (32) where $\beta$ can vary according to (28). A successful choice for $\beta$ (for the system used later on in the experiments) is shown in Fig. 6. If a $\beta$ can be found such that (33) holds, global asymptotic stability for the autonomous system is guaranteed, and thus step 1 of the proof. Given step 1 , appropriate boundedness criteria for step 2 ensure BIBO stability for the non-autonomous system. If an appropriate $\beta$ cannot be found, a quadratic Lyapunov function (with a constant $P$ ) guaranteeing stability does not exist for this system, thus different conditions need to be considered.

\section{Measurements}

The resonance feedforward control scheme presented in section III is validated using a rotational two-mass-springdamper system which is controlled in discrete-time, at sampling rate $F_{s}=1048 \mathrm{~Hz}$, and which is shown in Fig. 7. The discrete-time implementation is straightforward and will not further be explained due to space considerations. The inputoutput response of the LTI system consists of the collocated transfer function,

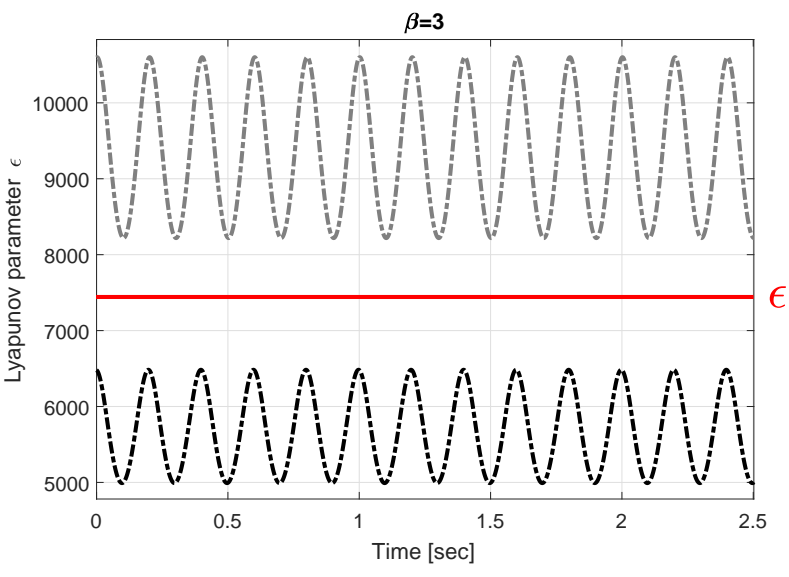

Fig. 6: Graphical check of stability for the system used in measurements of Section $\mathrm{V}$, for $\beta=3$. The grey line denotes $\delta_{1}(\beta, t)$, the black line $\delta_{2}(\beta, t)$, and the red line a valid $\epsilon$ which guarantees stability.

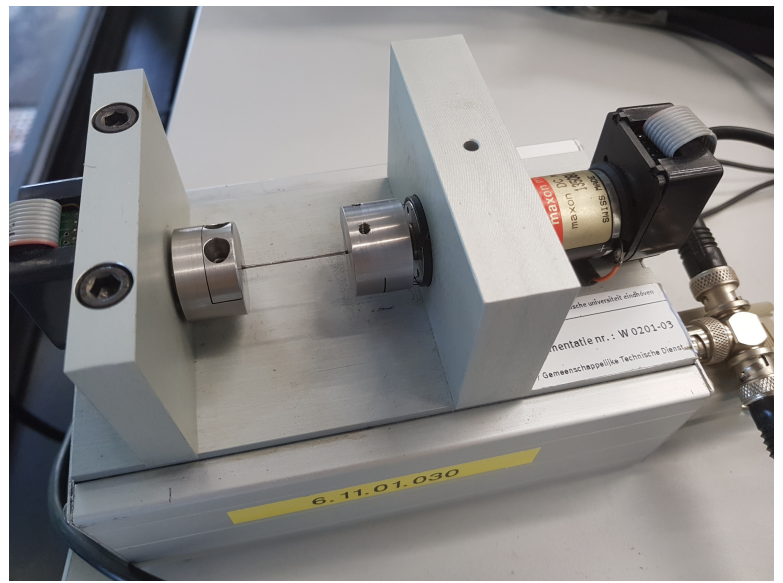

Fig. 7: Photo of the rotational two-mass-spring-damper (MSD) experimental setup used for measurements. The sampling rate of the encoders is set to $1048 \mathrm{~Hz}$.

$$
\mathcal{H}_{\mathrm{c}}(s)=\frac{x_{1}(s)}{F(s)}=\underbrace{\frac{1}{I^{\prime} s^{2}}}_{\mathcal{P}_{\mathrm{rb}}}+\underbrace{\frac{I_{2}^{\prime 2}}{I^{\prime}\left(I_{1}^{\prime} I_{2}^{\prime} s^{2}+d I^{\prime} s+I^{\prime} k\right)}}_{\mathcal{P}_{\mathrm{c}}},
$$

and the non-collocated transfer function,

$$
\mathcal{H}_{\mathrm{nc}}(s)=\frac{x_{2}(s)}{F(s)}=\underbrace{\frac{1}{I^{\prime} s^{2}}}_{\mathcal{P}_{\mathrm{rb}}}+\underbrace{\frac{-I_{1}^{\prime} I_{2}^{\prime}}{I^{\prime}\left(I_{1}^{\prime} I_{2}^{\prime} s^{2}+d I^{\prime} s+I^{\prime} k\right)}}_{\mathcal{P}_{\mathrm{nc}}},
$$

where $k=3.925[\mathrm{~N} \cdot \mathrm{m} / \mathrm{rad}], d=6.84 \cdot 10^{-4}[\mathrm{~N} \cdot \mathrm{m} \cdot \mathrm{s} / \mathrm{rad}]$. The constants $I_{1}^{\prime}, I_{2}^{\prime}$, and $I^{\prime}=I_{1}^{\prime}+I_{2}^{\prime}$ include both the moments of inertia of the two masses $I_{1}, I_{2}$, and the torque constant of the motor $K_{T}$, as follows, $I_{1}^{\prime}=I_{1} K_{T}=1.938$. $10^{-4}\left[\mathrm{~kg} \cdot \mathrm{I}^{3} \cdot \mathrm{N} / \mathrm{A}\right], I_{2}^{\prime}=I_{2} K_{T}=1.504 \cdot 10^{-4}\left[\mathrm{~kg} \cdot \mathrm{m}^{3} \cdot \mathrm{N} / \mathrm{A}\right]$ . From (35) it can be seen that $\mathcal{P}_{\text {rb }}$ denotes the RB mode, $\mathcal{P}_{\mathrm{c}}$ the collocated NRB mode, and $\mathcal{P}_{\text {nc }}$ the non-collocated NRB mode. 
An LTV system is created by gradually shifting between the collocated and the non-collocated outputs, as follows

$$
x_{\mathrm{out}}=r_{p}(t) x_{1}(t)+\left(1-r_{p}(t)\right) x_{2}(t), \quad r_{p}(t) \in[0,1],
$$

with $r_{p}(t)$ the POI function

$$
r_{p}(t)=0.5-0.4 \cos (10 \pi t)
$$

This leads to the LTV system

$$
\mathcal{H}(p)=\underbrace{\frac{1}{I^{\prime} p^{2}}}_{\mathcal{P}_{\mathrm{rb}}}+\underbrace{\frac{I_{2}^{\prime}\left(r_{p}(t) I^{\prime}-I_{1}^{\prime}\right)}{I^{\prime}\left(I_{1}^{\prime} I_{2}^{\prime} p^{2}+d I^{\prime} p+I^{\prime} k\right)}}_{\mathcal{P}_{\text {NRB-LTV }}} .
$$

The compliance function is given by $\mathcal{P}_{\text {NRB-LTV }}$ in (38), as follows,

$$
C\left(r_{p}(t)\right)=\left.\mathcal{P}_{\mathrm{NRB}-\mathrm{LTV}}\right|_{p=0}=\frac{I_{2}^{\prime}\left(r_{p}(t) I^{\prime}-I_{1}^{\prime}\right)}{I^{\prime 2} k} .
$$

The POI function was chosen to oscillate at $5[\mathrm{~Hz}]$, which poses enough challenge to the system such that the LTV performance of the feedforward controller can become apparent. For the same reason, the feedback controller $\mathcal{C}_{\mathrm{fb}}$ is chosen such that the bandwidth does not exceed $5[\mathrm{~Hz}]$, while stabilizing (38), treating the RB mode as the nominal system and the NRB-LTV mode as an additive uncertainty. The feedback controller consists of $\mathcal{C}_{\mathrm{PD}}$ which includes a PD controller with a second-order roll-off filter, $\mathcal{C}_{\text {I }}$ which adds integral action, and a notch filter $\mathcal{C}_{\mathrm{N}}$, given as follows,

$$
\begin{aligned}
\mathcal{C}_{\mathrm{fb}}(s)= & \left(\mathcal{C}_{\mathrm{PD}}(s)+\mathcal{C}_{\mathrm{I}}(s)\right) \mathcal{C}_{\mathrm{N}}(s)=(\underbrace{\frac{1.42 \cdot 10^{4} s+5.685 \cdot 10^{5}}{s^{2}+2513 s+1.58 \cdot 10^{6}}}_{\mathcal{C}_{\mathrm{PD}}(s)} \\
& +\underbrace{\frac{5.7}{s}}_{\mathcal{C}_{\mathrm{I}}(s)})(\underbrace{\frac{2.15 \cdot 10^{-5} s^{2}+1.41 \cdot 10^{-4} s+1}{2.15 \cdot 10^{-5} s^{2}+5.94 \cdot 10^{-4} s+1}}_{\mathcal{C}_{\mathrm{N}}(s)})_{(40)}
\end{aligned}
$$

The measurement results are shown in Fig. 8, where the tracking error using acceleration feedforward control, i.e.

$$
\mathcal{C}_{\mathrm{acc}}(s)=m s^{2}=3.442 \cdot 10^{-4} s^{2}
$$

is compared to the proposed resonance LTV feedforward controller as in (11) using the system specifications mentioned. It can be seen that the error of the proposed feedforward controller is much smaller. It is important to note here that the POI function and the feedback controller were especially chosen to illustrate this difference. More specifically, the POI function was chosen fast enough to simulate highly varying LTV dynamics. In turn, the feedback controller was chosen slow enough, i.e. to have a bandwidth as low as $5 \mathrm{~Hz}$. Consequently, frequencies near and above $5 \mathrm{~Hz}$ are amplified due to the waterbed effect. The POI function was chosen as a sinusoid of $5 \mathrm{~Hz}$ to exploit that fact. The reason this was necessary is that while in simulations the LTV feedforward controller provided always improved results by many orders of magnitude, these errors are usually too small to be

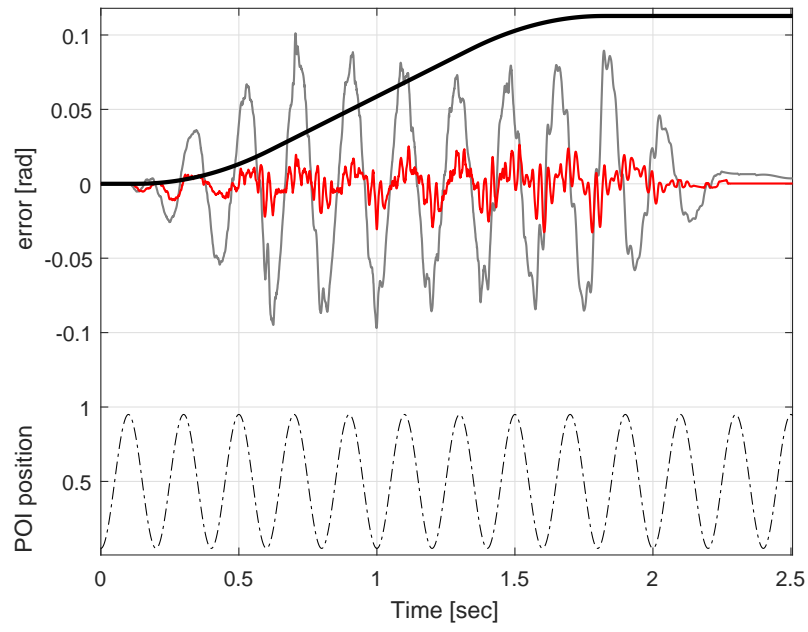

Fig. 8: Error with mass feedforward (grey), LTV feedforward (red), scaled set-point (solid black), and POI function (dashdot black).

detected by the encoders of the setup, and as such become invisible in the presence of measurement and quantization noise. The acceleration feedforward results indicate the error magnitude the LTV dynamics of this system can normally cause. From the tracking error of the LTV feedforward, it can be seen that this scheme can cope successfully with highly time-varying dynamics. Nonetheless, a residual error remains, which has two main components when analyzed through a cumulative power spectral density (CPSD) plot. The first residue comes from the POI function frequency itself, i.e. $5 \mathrm{~Hz}$, and the second component contains two frequency modulations of the main resonance at $34 \mathrm{~Hz}$, at $29 \mathrm{~Hz}$ and $39 \mathrm{~Hz}$, which indicates that the modulation is caused again by the POI function.

\section{CONCLUSIONS AND REMARKS}

This paper presents a controller which accounts for resonant and position-dependent compliant dynamics of LTV flexible plants. More precisely, a plant-inversion method is proposed using a fourth-order approximation model which captures the full compliance and the resonant dynamics of one of the NRB modes of the plant. Under appropriate conditions, which are graphically verifiable, the feedforward produces a feasible and bounded control signal. Measurement results using a two-mass-spring-damper system show the controller's ability to capture highly time-varying dynamics. This shows that the controller can produce feedforward signals which can successfully account for plant dynamics when using aggressive motion profiles and/or lightweight motion systems.

\section{ACKNOWLEDGMENTS}

The authors would like to acknowledge Karthik Subramanian Balasubramanian for his work in simulations using the proposed feedforward controller. 


\section{REFERENCES}

[1] Pao, L. Y., Butterworth, J. A., and Abramovitch, D. Y. (2007). "Combined feedforward/feedback control of atomic force microscopes", American Control Conference, pp. 3509-3515.

[2] Butterworth, J. A., Pao, L. Y., and Abramovitch, D. Y. (2012). "Analysis and comparison of three discrete-time feedforward model-inverse control techniques for nonminimum-phase systems", Mechatronics, Vol 22, No. 5, pp. 577-587.

[3] Yazaki, Y., Fujimoto, H., Sakata, K., Hara, A., and Saiki, K. (2015). "Application of mode switching control using initial state variables in constraint final-state control to high-precision dual stage", Proceedings of the American Control Conference, pp. 4155-4161.

[4] Devasia, S., Chen, D., and Paden, B. (1996). "Nonlinear inversionbased output tracking", IEEE Transactions on Automatic Control, Vol. , No. 7, pp. 930-942.

[5] Lambrechts, P., Boerlage, M., and Steinbuch, M. (2005). 'Trajectory planning and feedforward design for electromechanical motion systems", Control Engineering Practice, Vol. 13, No. 2, pp. 145-157.

[6] Ronde, M., van den Bulk, J., van de Molengraft, M.J.G.R., and Steinbuch, M. (2013). "Feedforward for flexible systems with timevarying performance locations", in American Control Conference, 2013, vol. 23, no. 4, , pp. 6033-6038.

[7] Kasemsinsup, Y., Heertjes, M., Butler, H., and Weiland, S. (2016). "Exact Plant Inversion of Flexible Motion Systems with a TimeVarying State-To-Output Map", European Control Conference, pp. 2483-2488.

[8] Chen, S.-L., Li, X., Teo, C.S., and Tan, K.K. (2017). "Composite jerk feedforward and disturbance observer for robust tracking of flexible systems", Automatica, Vol. 80, pp. 253-260.

[9] Kontaras, N., Heertjes, M.F., Zwart, H., and Steinbuch, M. (2017). "A compliance feedforward control design for position-dependent flexible systems", IEEE Transactions on Control Systems Technology, submitted.

[10] Zhou, B. (2016). "On asymptotic stability of linear time-varying systems", Automatica, Volume 68, June, Pages 266-276. 